\title{
Serological evidence of vector and parasite exposure in Southern Ghana: the dynamics of malaria transmission intensity
}

Kingsley Badu*, Ben Gyan', Maxwell Appawu², Daniel Mensah', Daniel Dodoo', Guiyun Yan³ , Chris Drakeley4, Guofa Zhou ${ }^{3}$, Ellis Owusu-Dabo ${ }^{5}$ and Kwadwo Ansah Koram ${ }^{6}$

\begin{abstract}
Background: Seroepidemiology provides robust estimates for tracking malaria transmission when intensity is low and useful when there is no baseline entomological data. Serological evidence of exposure to malaria vectors and parasite contribute to our understanding of the risk of pathogen transmission, and facilitates implementation of targeted interventions. Ab to Anopheles gambiae salivary peptide (gSG6-P1) and merozoite surface protein one $\left(\mathrm{MSP}{ }_{19}{ }_{19}\right.$ reflect human exposure to malaria vectors and parasites. This study estimated malaria transmission dynamics using serological evidence of vector and parasite exposure in southern Ghana.
\end{abstract}

Methods: Total IgG responses to both antigens in an age stratified cohort $(<5,5-14,>14)$ were measured from South-eastern Ghana. 295 randomly selected sera were analyzed from archived samples belonging to a cohort study that were followed at 3 consecutive survey months $(n=885)$; February, May and August 2009. Temporal variations in seroprevalence of both antigens as well as differences between the age-stratified cohorts were determined by $x^{2}$ test with $p<0.05$ statistically significant. Non-parametric repeated ANOVA - Friedman's test was used to test differences in antibody levels. Seroprevalence data were fitted to reversible catalytic model to estimate sero-conversion rates.

Results: Whereas parasite prevalence was generally low $2.4 \%, 2.7 \%$ and $2.4 \%$ with no apparent trends with season, seroprevalence to both gSG6-P1 and MSP1 ${ }_{19}$ were high $(59 \%, 50.9 \%, 52.2 \%)$ and $57.6 \%, 52.3 \%$ and $43.6 \%$ in respective order from Feb. to August. Repeated measures ANOVA showed differences in median antibody levels across surveys with specific significant differences between February and May but not August by post hoc Dunn's multiple comparison tests for gSG6-P1. For MSP1 ${ }_{19}$, no differences were observed in antibody levels between February and May but a significant decline was observed from May to August. Seroconversion rates for gSG6-P1 increased by 1.5 folds from February to August and 3 folds for MSP1 19 .

Conclusion: Data suggests exposure to infectious bites may be declining whereas mosquito bites remains high. Sustained malaria control efforts and surveillance are needed to drive malaria further down and to prevent catastrophic rebound. Operational factors for scaling up have been discussed.

Keywords: Anopheles gambiae, Salivary peptide, Malaria exposure, Seroprevalence, Seroconversion rate, Merozoite surface protein, Parasite prevalence

\footnotetext{
* Correspondence: Kingsbadu@gmail.com

'Department of Immunology, Noguchi Memorial Institute for Medical Research, College of Health Science University of Ghana, LG581, Legon, Accra, Ghana

Full list of author information is available at the end of the article
} 


\section{Background}

Malaria elimination has again been accepted as the goal of malaria control efforts with thirty-two out of the remaining ninety-nine malaria endemic countries currently implementing malaria elimination strategies [1]. As international efforts towards malaria elimination increase, accurate data on transmission intensity will be crucial for directing control efforts, developing and testing new control tools, and predicting the effects of these interventions under various conditions [2]. It has been suggested that during the pre-elimination phase, the focus of monitoring and evaluation of impact of interventions must shift from surveying health system indicators such as number of malaria cases and associated mortality to measuring malaria transmission intensity and infection [3-5]. However, very low malaria transmission intensity and the non-uniform transmission occurring after periods of extensive control [6,7] highlights important limitations associated with the current tools for measuring malaria transmission intensity. The lack of sensitivity of current malaria transmission tools constitutes a major bottleneck for malaria elimination efforts $[3,5]$.

The entomological inoculation rates (EIR), the product of man biting rates $(M a)$ and the sporozoite rate $(S R$, proportion of mosquitoes carrying sporozoites), is the gold standard for measuring malaria transmission intensity. It is the most direct way of detecting human exposure to infectious bites and mosquito population monitoring. However under conditions of very low malaria transmission the EIR suffers from well recognized limitations [2]. Notably, the intrinsic uncertainty in measuring $M a$ with methods such as human landing catches, resting collections, pyrethrum spray catches, and Centers for Disease Control and Prevention (CDC) light traps are all subject to operator-related variability, such that results may not be reproducible or accurately reflective of the overall local population, and the need for standardized methods for measuring both $M a$ and $S R$ [8,9] limit the precision and accuracy of EIR and its potential for measuring a change in transmission. This is especially so at low transmission intensities, where it is difficult to catch sufficient mosquitoes. The limitations associated with measuring malaria transmission by vector mosquitoes are expected to become even more pronounced as ongoing implementation of available control methods, including indoor residual spraying (IRS) and insecticide-treated nets (ITNs), drive down mosquito and malaria endemicity levels [10].

Parasite prevalence (PR), is a well-known metric that is used to estimate the proportion of the human population who are found to be carrying parasites in their blood [11]. The accuracy of outcome varies with the method used [12]. However, it generally becomes less reliable as a tool for measuring the intensity of malaria transmission when parasitemia is low [13]. As a result, more sensitive and standardized metrics are needed to assess transmission intensity in real time, to assess interventions, to acquire data necessary for planning appropriate control programs in areas of low transmission $[13,3]$.

Immuno-epidemiological assays based on human humoral responses to P. falciparum and Anopheles antigens are potentially valuable for robust transmission measurement [12-15]. In particular, the Merozoite Surface Protein 1 (MSP $1_{19}$ ) seroconversion rates has been shown to correlate with malaria transmission intensity (EIR), and to depict malaria endemicity by identifying hotspots of higher malaria transmission [15-18]. MSP$1_{19}$ seroprevalence and antibody level has proven to be sensitive in discriminating small spatial scales in malaria exposures at varying altitudes, age groups, and distance to Anopheles breeding habitats $[14,19,20]$.

The use of antibodies to Anopheles salivary proteins as a proxy for human exposure to vector bites and risk of parasite transmission is a promising endeavor. This phenomena rests on the concept that Anopheles vectors injects salivary proteins containing a cocktail of bioactive compounds including vasodilators and anticoagulants [21], which mitigate vertebrate host's defense mechanism such as hemostais, inflammation and thus facilitate blood feeding [22]. Some of the components of the bioactive compounds are antigenic and, elicits adaptive humoral response in the vertebrate host. The level of human exposure to Anopheles bites, have thus been found to correlate with the level humoral response to anti-salivary proteins [23,24]. This assay has so far been applied as an epidemiological marker of vector exposure and risk of pathogen transmission in exposed populations. So far, the utility of this application has been demonstrated in leishmaniasis [25], Chagas disease [26] and recently in malaria from western Kenya and elsewhere [20,24-26]. Due to the logistical difficulty in extracting whole saliva from mosquitoes and the possible cross reactivity between common epitopes within the dipteral group the recombinant protein (gSG6) specific to the Anopheles genus was isolated and purified for the assay [27-29]. Recently a synthetic peptide, the gambiae salivary gland peptide 1 (gSG6-P1) based on the recombinant protein with an enhanced Anopheles specificity and antigenecity has been developed and validated [20,30]. The synthetic peptide has standardized the assay and guaranteed high reproducibility such that it is possible to compare results from one lab to the other and from one region to the other. Antibody reactivity to this peptide shows promising characteristics as a biomarker for human biting by Anopheles mosquitoes. So far increases in gSG6-P1 specific antibody levels correlated with increased rainfall in a region of very low mosquito exposure and rapid decreases in these levels were observed in 
individuals after ITNs were introduced in areas of high malaria transmission [31,32]. The gSG6-P1 marker appears to have several characteristics of an ideal biomarker; firstly its very specific to the Anopheles genus with no relevant cross-reactivity with epitopes from other proteins or vectors of protozoan parasites [30,32]. Its synthetic nature largely ensures high reproducibility of the assay and it induces specific host humoral response which correlates with the level of exposure to An. gambiae bites.

We explored the utility of the Anopheline salivary peptide (gSG6-P1) in comparison with MSP-1 $1_{19}$, a well known malaria antigen, to examine the fine temporal variations in vector and parasite exposure in an area of low malaria transmission but high vector exposure. The ability to detect temporal changes in malaria transmission intensity will enable us to sensibly deploy scarce resources in a targeted focal control to yield maximum community or country benefits and speed malaria elimination.

\section{Methods}

Archived plasma samples for the current study were obtained from an earlier cohort study conducted in Asutuare and its surrounding areas. The cohort was selected from a relatively small geographic area within $5 \mathrm{~km}$ radius with non-significant differences in malaria exposure, and malaria antibody genetic markers described in detail by Adu et al. [33]. Thus, malaria variation in the cohort, in respect of geographic location was considered homogeneous. Asutuare is a sub-district of Shai Osudoku district, (formerly Dangbe west district) in Greater Accra of Ghana. It is a semi-rural area about $40 \mathrm{~km}$ northeast of Accra, the capital of Ghana. The district has a surface area of $1,442 \mathrm{~km}^{2}$ and a population size of 122,836 (population and housing census, 2010 (http://www.ghanadistricts.com/pdfs/2010_pop_census_ districts.pdf). The population is typically scattered in small satellite villages of about two thousand people. The district usually has two rainy seasons in a year, beginning from April to July and October to December. Malaria transmission is low but perennial, and peaks slightly during and immediately after the major rainy seasons and is lowest during the dry seasons [34]. It is estimated that individuals near the district capital are exposed to about 7.5 infective bites in the rainy season (Personal communication), and $98 \%$ of the infections are due to P. falciparum [34]. Historically there was a huge sugar processing company with corresponding commercial farms of sugar cane. The community thus has a network of canals created for irrigation schemes. This potentially exposes inhabitants to mosquito bites all year round. It is not surprising that the area has been a site for piloting malaria related interventions since the 1990s [35]. Overall, the sum of 560 participants between the ages of one and thirty were enrolled for the original study in 2009; these were followed up in three cross-sectional community-based surveys to collect blood samples for laboratory analysis; in February towards the end of the dry season, May near the peak of rainy season and August representing snap shots of the perennial transmission in the year. For the purpose of this study, sera from 295 subjects were randomly chosen and assayed from each time point.

\section{Sample size}

Sample size was determined by using the binomial model to estimate the confidence interval (CI) [36]. Because antibody prevalence to the salivary gland protein and the MSP1 were unknown in the area, the antibody prevalence of CSP (25\%) previously reported in the cohort [37] was used as the antibody prevalence to estimate the sample size. The sample size with a 95\% CI and precision level of 5\% was estimated according to the formulae below: In this equation, $n$ is the sample size, $z$ is the critical value of the standard normal distribution at the $5 \%$ level (1.96), $p$ is the prevalence of antimalarial CSP antibody, $q=1-p$, and $d$ is the precision level. The population size was estimated to be 2,000 . The minimum sample size was estimated to be 288 .

$$
n_{o}=\frac{Z_{a} P q}{d^{2}}
$$

\section{Ethical approval}

The study used sera samples archived from an earlier cohort study conducted in 2009.

Ethical clearance for the main study was granted by the Institutional Review Board (IRB) of the Noguchi Memorial Institute for Medical Research (NMIMR). Study participants or their parents/legal guardians gave written informed consent, which permitted storage for future malaria studies, before enrolment into the study. For the present study, analysis of archived sera was blinded.

\section{Anopheles gambiae salivary antigen}

Bioinformatic tools were used to design and optimize the specificity and immunogenicity of the Anopheles gambiae salivary peptide (gSG6-P1) as previously reported $[30,20]$. Protein sequences were sent to Genepep (St-Jean de Vedas, France) which synthesized and purified (>95\%) the peptide. This was then shipped to Ghana in lyophilized form.

\section{Testing for Total IgG antibodies to An. gambiae antigen (gSG6-P1)}

Serologic testing of human exposure to gSG6-P1 was achieved using Enzyme-Linked Immunosorbent Assay 
(ELISA) as previously reported [20]. Briefly, gSG6-P1 peptide $(20 \mu \mathrm{g} / \mathrm{mL})$ was used to coat Maxisorp microtitre plates (Roskilde, Denmark) and incubated at $37^{\circ} \mathrm{C}$ for $2 \frac{1}{2} \mathrm{hrs}$ and then washed. Blocking buffer $(0.5 \%$ Casein $0.05 \%$ Tween 20) was added for $1 \mathrm{hr}$ at room temperature. Sera from participants were diluted at an optimized dilution of 1:20 diluent, and kept at $4^{\circ} \mathrm{C}$ over night. Polyclonal goat anti-human IgG antibody conjugated to horse radish Peroxidase (Nordic Immunology, Tilburg, Netherlands) at a dilution of 1:10,000 in PBS was used to detect Anti-gSG6- P1 IgG. A peroxidase substrate ABTS (Kirkegaard \& Perry Laboratories Inc., Gaithersburg, MD) was then added after washing and kept at room temperature for 50 mins. Enzymatic reaction was stopped with $4 \mathrm{~N} \mathrm{H}_{2} \mathrm{SO}_{4}$. Optical density (OD) was then read at $450 \mathrm{~nm}$ on a spectrophotometer. Test sera were evaluated in duplicates with a corresponding third well containing no antigen (ODn), a blank well to control for non-specific reactions in the sera and the reagents. IgG levels were determined as final OD computed for each serum as the mean OD value (with antigen) minus the OD value without antigen (ODn-blank well). For the purposes of quality assurance, intra- and inter-assay disparity of control samples was below $20 \%$. Sera whose duplicates had a coefficient of variation (CV) $20 \%$ and above were excluded from the analysis. The mean OD of unexposed controls (from the Europe, $\mathrm{N}=30$ ) plus $3 \mathrm{SD}$ was used as cut-off value for seropositivity.

\section{Measurement of anti-human IgG antibodies for $P F M S P 1_{19}$ (FVO) antigen}

Total IgG antibody to $P f \mathrm{MSP} 1_{19}$ was measured by indirect ELISA as previously described [14]. The expression and purification of the $P f M S P 1_{19}$ recombinant protein has also been described [14,20]. Assay plates (Maxisorp, Roskilde, Denmark) were coated with $0.5 \mu \mathrm{g}$ PfMSP1 recombinant protein and incubated overnight at $4^{\circ} \mathrm{C}$. After blocking, test sera were added in duplicate wells. HRP conjugate of goat antihuman IgG (KPL, Gaithersburg, MD) was added after incubation. Then ABTS (Kirkegaard \& Perry Laboratories Inc., Gaithersburg, MD) was added and incubated for $1 \mathrm{~h}$ at $22^{\circ} \mathrm{C}$. The OD measurements were taken at $414 \mathrm{~nm}$ on a spectrophotometer (SpectraMAX 340PC, Molecular Devices Corporation). Duplicate optical densities were averaged and normalized against a positive control. The cut off for seropositivity was an OD three standard deviations or more above the mean OD obtained in samples from 25 Europeans with no history of previous malaria exposure.

\section{Data analysis}

Seroprevalence was defined as the number of positive responders out of the total number of participants tested.
The temporal variations in seroprevalence of both antigens as well as differences between the age-stratified cohorts were determined by $\chi^{2}$ test with $\mathrm{p}<0.05$ considered statistically significant. The non-parametric repeated ANOVA - Friedman's test was used to test if median antibody levels were different across different survey months (Feb, May and August) as well as the different age strata. The Post Hoc Dunn's multiple comparison tests was used to test specific differences in seroprevalence between specified survey months and age groups. Age specific MSP- $1_{19}$ and gSG6-P1 seroprevalence data was fitted to a simple reversible catalytic model using the maximum likelihood method that assumes a binomial error distribution; $P t=\lambda /(\lambda+\rho)[1-\exp (-$ $\left.(\lambda+\rho)^{t}\right)$ ] where Pt is the proportion of individuals aged $t$ that is seropositive, Lambda $\lambda$ is the annual rate of seroconversion and $\rho$ is the annual rate of reversion to seronegative. This was done to investigate the relationship between force of vector and parasite exposure with age. All data were analysed and graphed using GraphPad Prism software (San Diego, CA, USA).

\section{Results}

\section{Study population and parasite prevalence}

A total of 295 subjects had data available for all the 3 surveys (Feb, May and Aug) and thus all analysis were based on the 295 selected samples. $27 \%$ out of the subjects were less than 5 yrs whilst the majority of the study subjects were between the ages of 5 and 14 . Only $12 \%$ of the participants in the selected cohort were in the in the age bracket of 15-29 (Table 1).

\section{Number of participants, parasite prevalence and antigen specific seroprevalence}

Parasite prevalence was generally low with no particular seasonal trends observed across the three contact months (Table 1). Within the age groups, parasite prevalence by microscopy rose from an average of $1.7 \%$ in < 5 year group to a two-fold increase (3.4\%) in the 5-14 year group but a complete absence $(0 \%)$ in the adult group. However, Chi square analysis revealed no significant differences in parasite prevalence when the three age groups were compared (Table 1).

\begin{tabular}{|c|c|c|c|c|c|c|}
\hline \multirow[t]{2}{*}{ Age } & \multirow[b]{2}{*}{ N (\%) } & \multicolumn{3}{|c|}{ Parasite prevalence (\%) } & \multirow[t]{2}{*}{$x^{2}$} & \multirow[t]{2}{*}{$P$ value } \\
\hline & & Feb & May & Aug & & \\
\hline$<5$ & $80(27)$ & 2.5 & 1.3 & 1.3 & 0.51 & 0.77 \\
\hline $5-14$ & $179(61)$ & 2.8 & 3.9 & 3.4 & 0.34 & 0.8 \\
\hline $15-29$ & $36(12)$ & 0.0 & 0.0 & 0.0 & ND & ND \\
\hline$x^{2}$ & & 1.01 & 2.63 & 2.05 & & \\
\hline$P$ value & & 0.60 & 0.27 & 0.36 & & \\
\hline
\end{tabular}


Table 2 Comparison of antigen specific seroprevalence at the population level in different survey months

\begin{tabular}{|c|c|c|c|c|c|}
\hline \multicolumn{4}{|c|}{ Antigen- specific Seroprevalence } & \multirow[t]{2}{*}{$x^{2}$} & \multirow[t]{2}{*}{$s$} \\
\hline & Feb & May & August & & \\
\hline gSG6-P1 & 59.8 & 50.9 & 52.2 & 4.18 & 0.123 \\
\hline PfMSP $1_{19}$ & 57.6 & 52.3 & 43.6 & 6.84 & 0.033 \\
\hline
\end{tabular}

\section{Antigen specific seroprevalence}

Exposures to the Anopheles gambiae salivary protein (gSG6-P1) were high throughout the three survey months, although a $10 \%$ decline in seroprevalence was observed between $1^{\text {st }}$ and $2^{\text {nd }}$ survey months (February and May), this was just at the threshold of statistical significance ( $\chi 2=3.6, D F=1, p=0.05)$. There was a significant but gradual decline in exposures to the blood stage $P$. falciparum merozoite surface proteins (MSP1 $1_{19}$.), $\mathrm{MSP}_{19}$ specific seroprevalence observed in $1^{\text {st }}$ survey declined by $5 \%$ in the $2^{\text {nd }}$ survey and a further $10 \%$ in August (Table 2).

\section{Correlation of antigen specific seroprevalence and parasite prevalence with age}

The spearman correlation analysis revealed an inverse relationship between $P$. falciparum prevalence and age for all three survey months although this was not strong to achieve statistical significance; Feb $(\mathrm{r}=-0.30 ; p=0.46)$, May $(\mathrm{r}=-0.05 ; p=0.93)$ and August $(\mathrm{r}=-0.39 ; p=0.32)$. High exposures to the salivary gland peptide corresponded to strong association with age observed in the first and second survey months (Figure 1), however these exposures gradually declined with a non significant correlation with age in the third survey $(\mathrm{r}=0.16 ; p=0.11)$. gSG6-P1 responses had very similar trends compared with that observed in $\mathrm{MSP}_{19}$ which also had a strong correlation in the first survey with a gradual decline in the second and third surveys (Figure 1).
Comparison of antigen-specific median antibody levels at the cohort population level

Median antibody levels of gSG6-P1 and MSP1 19 antigens as measured in optical densities in the cohort at all survey months across age groups were above the threshold of the unexposed group. Median levels of anti gSG6-P1 antibody showed significant differences in antibody levels in mosquito exposure between the first survey (February 2009) and second (May 2009) survey months, detecting temporal variations in vector exposure among the cohorts at different time points. The Friedman's test (Repeated measures ANOVA and non parametric) revealed significant differences in antibody levels to gSG6P1 between the first, second and third survey months at the population level. $(\mathrm{F}=33.97 ; p<0.0001)$ as well as post hoc Dunn's multiple comparison tests between Feb and May: $(p<0.0001)$, Feb and August $(p<0.0001)$, but not May and August $(p>0.5)$ (Figure 2).

Similar to the differences in gSG6-P1, the Friedman's statistic detected overall significant differences in anti $\mathrm{MSP}_{19}$ levels compared across survey months $(\mathrm{F}=14.98$; $p=0.0006)$. However, post hoc analysis revealed no significant differences between first and second (February and May) survey months $(p>0.05)$ but significant decline were observed in the antibody levels between the second and third (May-August) survey months $(p<0.05)$ (Figure 2).

\section{Age-stratified Cohort and Median antibody levels}

Generally, median antibody levels to all antigens differed among the age groups throughout the surveys (KruskalWallis $\mathrm{H}$ test) (Figure 3). However, post hoc Dunn's multiple comparisons test revealed differences between specific pairs of age groups.

Kruskal-Wallis $\mathrm{H}$ statistic revealed significant differences in anti gSG6-P1 level across all age-stratified cohorts and survey months; Feb $(\mathrm{H}=17.49 ; p=0.0002)$, May $(\mathrm{H}=11.09 ; \mathrm{p}=0.0039)$ and August $(\mathrm{H}=10.43$;

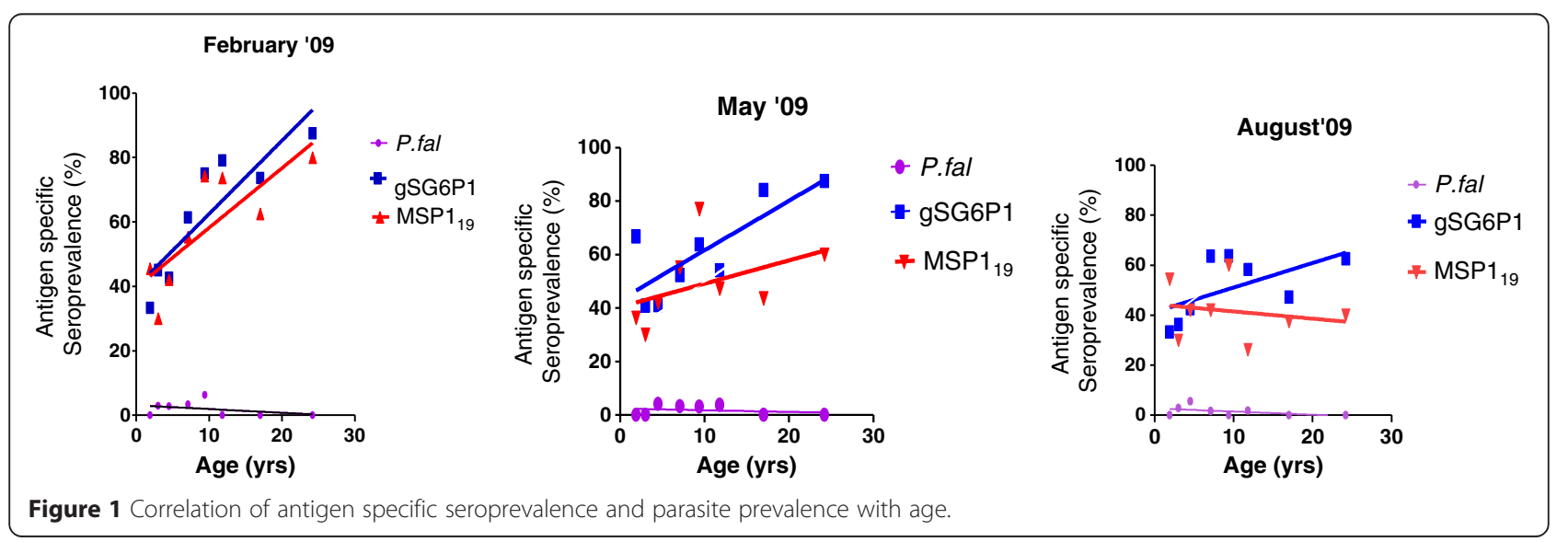



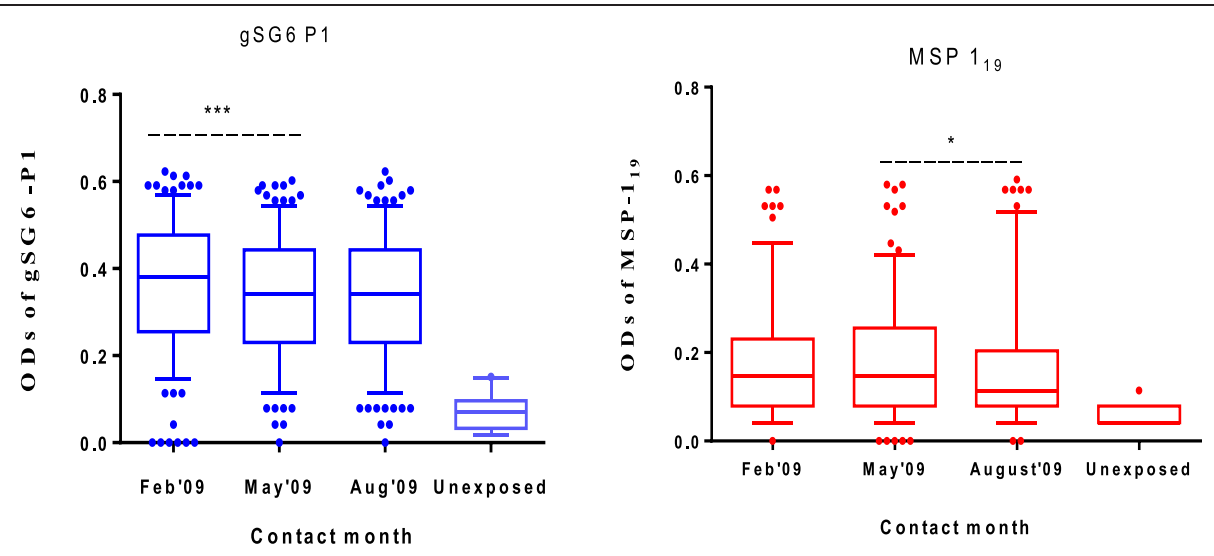

$* * *$ indicate $\mathrm{P}$ value $<0.0001, * *$ indicate $\mathrm{P}<0.01$ and * indicate $\mathrm{p}$ value $<0.05$

Figure 2 Comparison of median antigen specific antibody levels in within the study cohort.

$p<0.0054)$ with increasingly higher antibody levels. However, the Dunn's multiple comparison tests (post hoc test) between specific pairs of age-stratified cohorts showed intriguing differences: consistently, there were significant differences between $<4$ and the $5-14$ age groups across all survey months, Feb $(p<0.001)$ May $(p<0.01)$ and Aug $(p<0.01)$ with higher antibody levels observed in the 5-14 year cohorts. However, there were no differences seen in the antibody levels of anti-gSG6P1 in the 5-14 and the >14 age group in the first and second

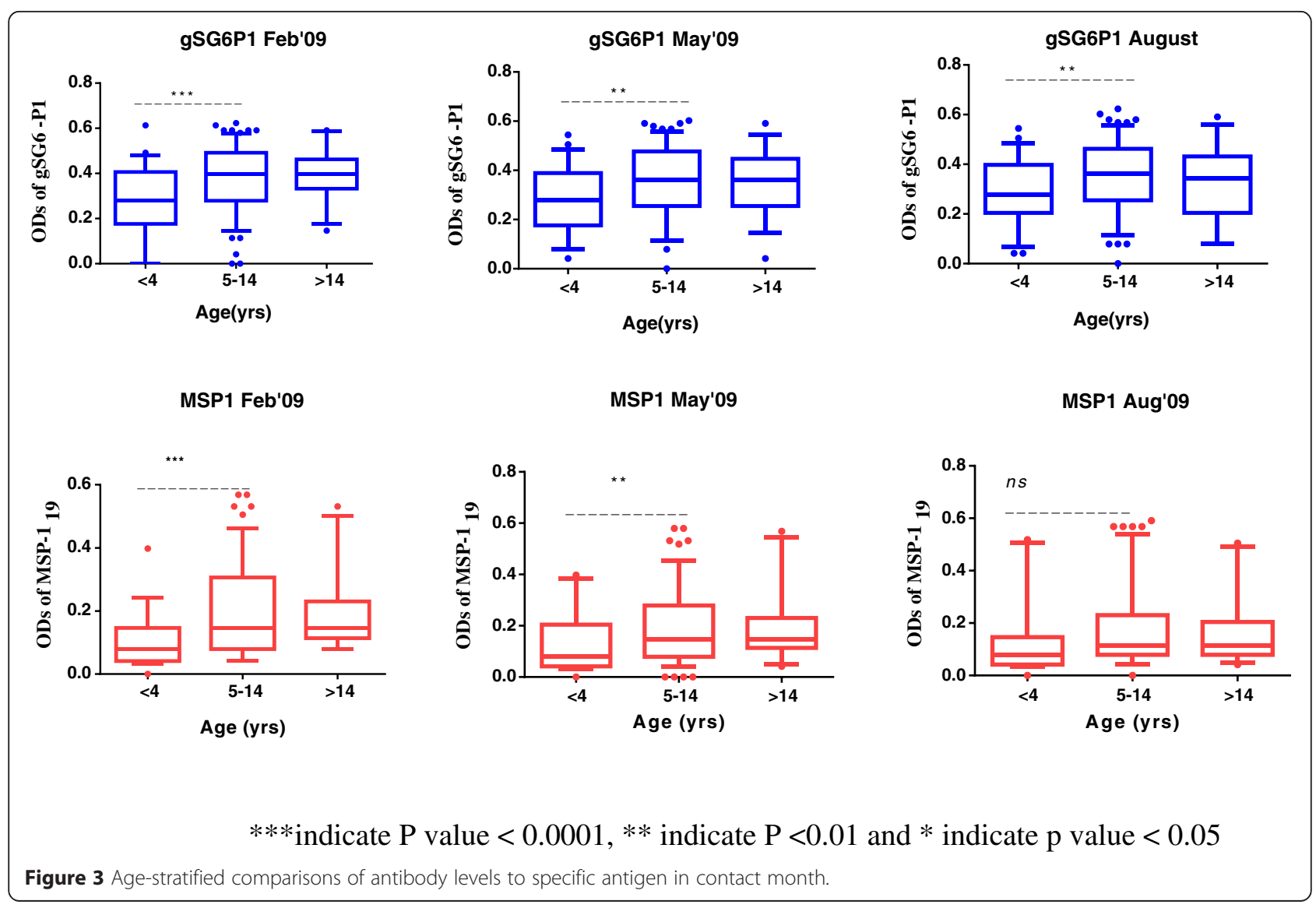


survey months $(\mathrm{P}>0.05)$, a decline in between the third survey with the $>15$ year cohort having lower gSG6-P1 levels but this was not statistically significant (Figure 3 ).

Median antibody levels to the merozoite surface protein $\left(\mathrm{MSP}_{19}\right)$ increased significantly between $<5$ and $5-14$ age groups in the first and second survey months but not the third (Figure 3). In respective order Feb $(\mathrm{H}=20.01$; $p<0.0001)$ May $(\mathrm{H}=9.43 ; p<0.01)$ August $(\mathrm{H}=4.99$; $p>0.05)$. Similar to gSG6-P1, there were no differences in the antibody levels observed between the 5-14 and the adult age-groups throughout the three survey months.

\section{The force of exposure to vector bites and parasite over time}

The force of exposure to both antigens (gSG6-P1(Figure 4) and $\mathrm{MSP}_{19}$ (Figure 5)) tested increased significantly with age at all contact months, however the rate at which individuals change from being seronegative to seropositive differed with each antigen and survey month, gSG6-P1(Figure 4), MSP1 19 (Figure 5) . Generally, seroconversion rates $(\lambda)$ (SCR) for gSG6-P1 and $\mathrm{MSP}_{19}$ gradually increased from February to August, The force of exposure to vector bites increased 1.5 fold from February to August (Figure 4). MSP1 19 SCR increased marginally between 1.5 folds to 3 folds from February to May then to August. Sero-reversion rates $(\rho)$, also recorded a somewhat corresponding increase (Figure 5).

\section{Discussion}

Metrics of malaria transmission change on different temporal scales, mirroring among other things the changes in vector exposure, parasite infections in humans, as well as the dynamics of changing human immunity. The key component in deciding the appropriateness of malaria transmission metrics as end points for measuring changes in transmission is determined not only by costs, precision, and accuracy, but also by the intrinsic variability of the metric across space and time [38]. Using age specific seroprevalence data, antibody density and seroconversion rates, it is possible to identify temporal variations in mosquito and parasite exposure to show the variation in the intensity of malaria transmission that would otherwise go undetected by parasite prevalence due to apparent low transmission in the Asutuare area.

The use of serological markers of vector and parasite exposure to track changes in malaria transmission over time has several competitive advantages over other metrics. Chiefly, serological markers are more sensitive and robust $[15,16,14]$. For equivalent parasite rates, serological markers generate seroprevalence that is higher than the parasite prevalence revealing active transmission in progress over a period of time that would otherwise be deemed as interrupted transmission [13,39]. In the current study whereas parasite prevalence was well below $5 \%$ throughout the year; equivalent seroprevalence of gSG6-P1 and MSP $1_{19}$ was $40 \%$ and above. Similar findings have been reported elsewhere. In Somalia, when no parasites were detected both in wet and dry seasons after screening more than one-thousand people, $17.9 \%$ and $19.3 \%$ of $\mathrm{MSP}_{19}$ seroprevalence was found respectively [13]. Similarly, in the uphill dwellers of the Western Kenyan highlands it is reported that when parasite prevalence was well below 10\%, seroprevalence of gSG6$\mathrm{P} 1$ and $\mathrm{MSP}_{19}$ reached peaks above 50\% [20].

Serological markers, in particular gSG6-P1, are robust in tracking temporal changes in vector exposure and risk of pathogen transmission in the younger population under intense transmission intensity $[20,27,28,31,32,40]$. In the adult population however, there seem to be an immuno-tolerance to the recombinant protein version (gSG6) where higher exposures result in declining immune response, this has been explained as desensitization of the salivary proteins to the immune system of the adults [41]. It is known to be highly antigenic in naïve individuals with only transient exposure and wanes rapidly in the absence of continuous exposure, this has variously been corroborated in Senegal and Angola [31,42,43]. Sagna et al. studied human immune response to gSG6-P1 salivary peptide in five communities in northern Senegal where malaria transmission has been described as low, with the An. gambiae s. $l$. as the principal vector. They
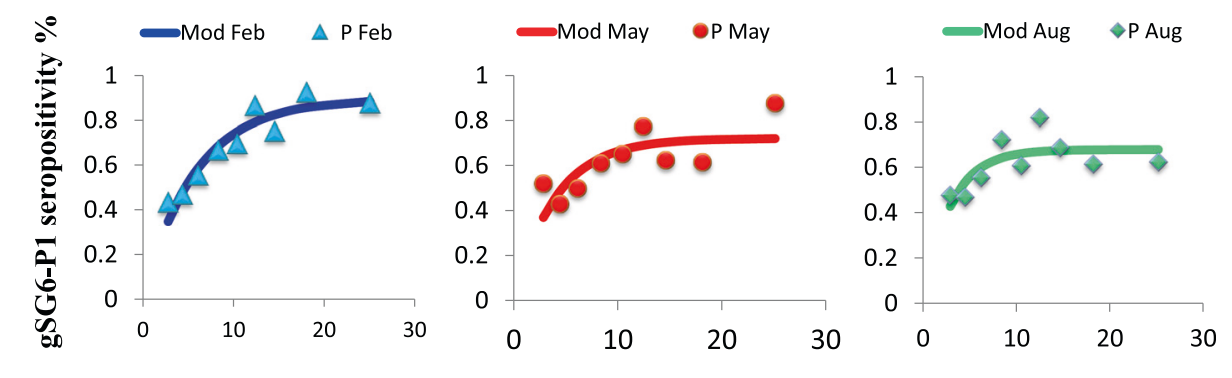

Participant age (years)

Figure 4 Seroconversion rate gSG6-P1. 

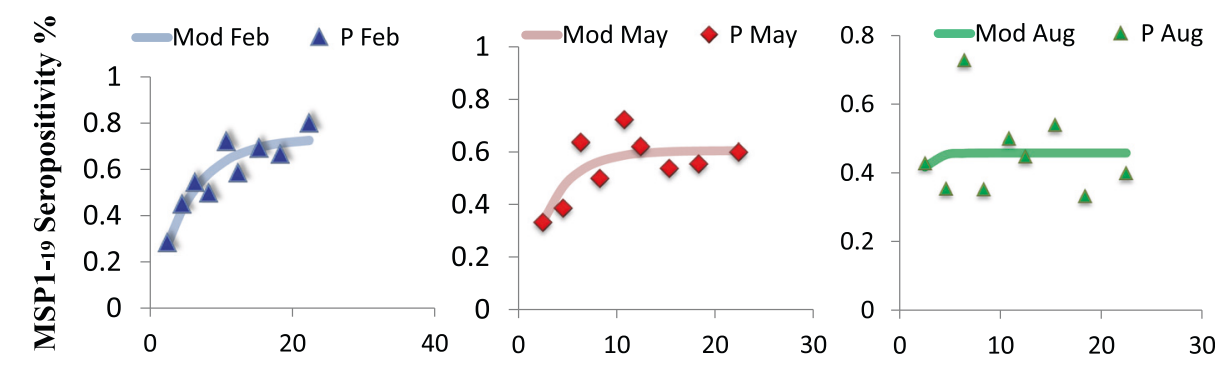

Participant age (years)

Figure 5 Seroconversion rate $M S P 1_{19}$.

observed a significant increase in IgG levels to gSG6-P1 during the peak exposure to Anopheles bites, and a corresponding decrease right after the end of the exposure season [31]. Moreover, IgG levels to gSG6-P1 varied considerably according to the villages, discriminating the heterogeneity of Anopheles exposure between villages [20,31].

In the current study it was observed that Total IgG anti-gSG6-P1 levels showed significant differences between February and May but showed sustained high levels in August. Asutuare is a sugar-cane growing area; a cash crop that thrives in water logged areas, thus the area has several irrigation schemes that virtually flood the area all year round exposing inhabitants to high vector exposure. High vector exposure as evidenced from the anti-gSG6-P1 responses was observed in the month of February, a relatively dry month, this may be due to lagged effect of high rainfall recorded two-three months earlier in 2008 [37]. Meteorological variables have distinct patterns and effects on malaria transmission due to specific lagged correlations and most time series studies have provided evidence of an association between rainfall and mosquito abundance, typically at a single lag of 0 , 1 or 2 months depending on the mosquito species [44]. The Anopheles gambiae, a principal malaria vector in the district, requires relatively shallow and transient breeding aquatic habitat and therefore its abundance does not peak immediately after heavy rainfall but must tarry for the flooding waters to recede.

$\mathrm{MSP}_{19}$ Age-specific seroprevalence has been used to estimate seroconversion rates (SCR) as a measure of malaria transmission intensity. Earlier studies in Tanzania have shown that these estimates are tightly correlated with EIR measurement $[15,16,45]$. Age seroprevalence curves reflect different levels of transmission intensity. In low transmission settings development of antibodies is slow and prevalence is higher by the adult population, whereas in a high transmission area, much of the population will be seropositive even at a younger age [18]. The SCR of parasite exposure based on $\mathrm{MSP}_{19}$ seroprevalence has been described as an equivalent of the force of infection and correlated with the entomological inoculation rate. This measures the rate of parasite exposure in the population with age (or time) $[15,16]$. In the current study the MSP $1_{19}$ SCR increased about 3 fold from February to August. Although seroprevalence decreased approximately $8 \%$ (gSG6-P1) and 14\% (MSP1 19 ) at the population level from the February to August (Table 2), the SCR is a function of age and exposure. It is noteworthy that, when the data was stratified into age cohorts, seroprevalence to both antigens increased significantly from the 1-4 to the 5-14 year groups in all survey months (Figure 3) except for $\mathrm{MSP}_{19}$ in August. Moreover, although parasite prevalence showed a non-significant decline from 2.7\% (May) to $2.4 \%$ (August), age stratifications revealed that parasite prevalence increased with age in the paediatric population which happens to be $88 \%$ of the total study population (Table 1). Parasite prevalence within the $>5$ year group increased from $2.5 \%, 1.3 \%$ and $1.3 \%$ in first (Feb), Second (May) and third (August) survey months to 2.8\%, 3.9\% and $3.4 \%$ respectively. Thus this finding confirms the inherent ability of the SCR to reflect changes in transmission in terms of exposure and time.

The force of exposure to vector bites as seen in seroprevalence and antibody levels in study participants increased 1.5 fold from February to August. This implies that the aggressiveness of vector bites increased or the Anopheles population density increased. It has been suggested that the gSG6-P1 is a reliable marker for measuring human-vector contact [32]. Possibly it depicts the human vector contact like the $m a$ measured in EIR estimation, this is because human immune response to gSG6-P1 increases and decreases sharply following high and low vector exposure seasons, and transient exposure in naïve individuals, this has been observed by other scientist elsewhere [32,42]. In Lobito, a malaria-endemic coastal city of Western Angola, Papa Drame and coworkers conducted a longitudinal evaluation of the efficient use of insecticide treated net (ITNs) using parasitological, entomological and immunological assessments in children 
as well as adults in 2010 [32,46]. A significant decrease in anti-gSG6-P1 IgG levels was observed just after the efficient use of ITNs with a subsequent rise in IgG levels to the peptide about four months after when the correct usage of ITN had waned. They thus concluded that the gSG6-P1 "provides a valuable tool in malaria vector control based on a real measurement of human-vector contact" It has also been observed to correspond with malaria endemicity at the population level discriminating villages with higher malaria exposure $[27,20,47]$. It is thus plausible to state that gSG6-P1 has the inherent ability to track changing vector exposure [32]. It has been shown that gSG6-P1 is able to detect differences in vector exposure in different age groups and distance from Anopheles breeding sites [20], rapid buildup and decay of antibody levels [42]. The particular usefulness of gSG6-P1 is its ability to show differences in the short term exposure as has been seen in this study.

Parasite prevalence (PR) in the current study was generally low, with no exposure in the adult population $>15$ years, this has been reported by other scientists working in the same area [44]. The prevalence was determined solely by microscopy which may have underestimated the prevalence [48]. Parasite prevalence generally tends to be lower in adult population due to their well-developed immunity [49]. However, the strength of the relationship between PR and age depends on the endemicity or the transmission intensity of the area. Similar to this study, no significant relationship was observed between PR and age in the uphill residents of western Kenyan highlands but with low malaria transmission intensities. However, there was a strong inverse relationship in the valley dwellers with relatively higher transmission intensity [14,50]. Under low transmission intensity, specifically when parasite prevalence is below $1-5 \%$, it is complicated to use PR as a metric to detect changes in transmission or evaluate impact of interventions [51]. This has been attributed to several factors associated with the PR. Fundamentally, PR is inherently imprecise because parasite densities fluctuate over the course of a single infection [38], again the accuracy (sensitivity and specificity) of parasite detection in an infected blood depends on the number of parasites/ $\mu$ of blood with the probability of detecting parasite decreasing at low densities. Both microscopy and RDTs fail to detect subpatent infections when the parasite density is less than 250 parasites per $\mu \mathrm{l}$ [12]. In addition, parasite densities are strongly affected by the recent history of antimalarial drug intake and parasite resistance to those drugs [15]. Thus parasite prevalence is only a discrete measure that represents a snap shot of complex dynamic interactions which may need intensive multiple sampling all year round to be accurate.
Seroprevalence and seroconversion rates of vector (gSG6-P1) and parasite proteins (MSP1 $1_{19)}$ provide a useful sero-surveillance tool for tracking malaria transmission intensity. In order to scale.

Up this approach, three major issues need to be considered: the source of antigens, collection of study sample and laboratory processing to generate seroprevalence data. The salivary gland antigen is a short sequence synthetic peptide that can be synthesized in-house or by any commercial peptide company. Its synthetic nature generates reproducible results that can be compared across different laboratories [14,30]. Collection of blood in sero-epidemiological surveys used to be very cumbersome especially when antibodies needed to be quantified. However, this has been simplified in recent times, since antibodies can be eluted from filter paper. This has made sample collection and storage uncomplicated [52]. Lastly, the Enzyme Linked-Immuno-sorbent Assay (ELISA) is high throughput, standardizable assay, which is cheap, easy to perform and also generates objective reproducible results. Tusting et al. [38], actually quantified the cost per sample with ELISA as USD $\$ 0.5$ and with a turnover of 1000 samples per week. Other methodologies like the multiplex suspended bead assay (Luminex ${ }^{\mathrm{Tn}}{ }^{\mathrm{N}}$ ) and protein microarrays [53] have the added advantage of analyzing several antigens per sample and require smaller amounts of sera however; these are relatively expensive due to the capital intensive equipments.

\section{Conclusion}

Using antibody levels to gSG6-P1 and MSP1 $1_{19}$, seroprevalence and seroconversion rates (SCR) together with parasite prevalence, we have identified low parasite prevalence, high vector exposure and small changes in malaria transmission intensity from February through May and then to August 2009. Seroconversion rates for gSG6-P1 increased by 1.5 folds from February to August and 3 folds for $\mathrm{MSP}_{19}$. Possibly, exposure to infectious bites may be declining whereas mosquito bites remains high. Malaria transmission in Asutuare as well as many other areas in Africa where endemicity is low needs careful monitoring with sensitive and robust tools to ascertain progress and to forestall a potential catastrophic rebound.

\section{Competing interest}

The authors declare that they have no competing interests.

\section{Authors' contribution}

$\mathrm{KB}, \mathrm{BG}, \mathrm{KAK}$ and $\mathrm{GY}$ conceived and designed the study; $K B, B G$ and $D M$ carried out the laboratory immunological assays and analysis. CD purified and provided recombinant $M S P 1_{19}$ antigens. KB and GZ carried out the statistical analysis. KB wrote the first draft, BG, DD, CD GY MA, EOD GZ and KAK participated in manuscript development. All authors made substantial intellectual input to merit authorship. All authors read and approved the final version of the manuscript. 


\section{Acknowledgements}

We would like to thank Mrs. Nana Oye Akuffo, Ms Pamela Nai and Mr Adams Kpebu for providing administrative assistance for this study. This study was supported by the Bill and Melinda Gates Foundation-Global Health funded Postdoctoral Training Programme at Noguchi Memorial Institute for Medical Research, University of Ghana. Our funders neither played any role in study design, collection of data its analysis nor decision to publish.

\section{Author details}

'Department of Immunology, Noguchi Memorial Institute for Medical Research, College of Health Science University of Ghana, LG581, Legon, Accra, Ghana. ${ }^{2}$ Department of Parasitology, Noguchi Memorial Institute for Medical Research, College of Health Science University of Ghana, LG581, Legon, Accra, Ghana. ${ }^{3}$ Program in Public Health, Room 3038, Hewitt Hall, College of Health Science, University of California, Irvine, CA 92697-4050, USA. ${ }^{4}$ London School of Hygiene and Tropical Medicine, London, UK. ${ }^{5}$ Kumasi Centre for Collaborative Research in Tropical Medicine, School of Medical Sciences, Kwame Nkrumah University of Science and Technology, Kumasi, Ghana. ${ }^{6}$ Department of Epidemiology, Noguchi Memorial Institute for Medical Research, College of Health Science University of Ghana, LG581, Legon, Accra, Ghana.

\section{Received: 12 January 2015 Accepted: 15 April 2015}

\section{Published online: 28 April 2015}

\section{References}

1. Feachem RGA, Phillips AA, Hwang J, Cotter C, Wielgosz B, Greenwood BM, et al. Shrinking the malaria map: progress and prospects. Lancet. 2010;376:1566-78. doi:10.1016/S0140-6736(10)61270-6.

2. James S, Takken W, Collins FH, Gottlieb M. Perspective piece: needs for monitoring mosquito transmission of malaria in a Pre-elimination world. Am J Trop Med Hyg. 2014;90:6-10. doi:10.4269/ajtmh.13-0175.

3. Alonso PL, Brown G, Arevalo-herrera M, Binka F, Chitnis C, Collins F, et al. A research agenda to underpin malaria eradication. PLoS Med. 2011;8:e1000406. doi:10.1371/ journal.pmed.1000406.

4. Bousema T, Kreuels B, Gosling R. Adjusting for heterogeneity of malaria transmission in longitudinal studies. J Infect Dis. 2011;204:1-3. doi:10.1093/ infdis/jir225

5. Bousema T, Griffin JT, Sauerwein RW, Smith DL, Churcher TS, Takken W, et al. Hitting hotspots: spatial targeting of malaria for control and elimination. PLoS Med. 2012;9:1-7. doi:10.1371/journal.pmed.1001165.

6. Rotllant G, Bhattarai A, Stro J, Kahigwa E, Garcia M, Petzold M, et al. Influence of rapid malaria diagnostic tests on treatment and health outcome in fever patients, Zanzibar - a crossover validation study. PLoS Med. 2009;6:e1000070.

7. Clark TD, Greenhouse B, Njama-meya D, Nzarubara B, Maiteki-sebuguzi C, Maiteki-Sebuguzi N, et al. factors determining the heterogeneity of malaria incidence in children in Kampala, Uganda. J Infect Dis. 2008;198:393-400. doi:10.1086/589778.

8. Hay SI, Rogers DJ, Toomer JF, Snow RW. Annual Plasmodium falciparum entomological inoculation rates (EIR) across Africa: literature survey, internet access and review. Trans R Soc Trop Med Hyg. 2000;94:113-27.

9. Kelly-hope LA, Mckenzie FE. The multiplicity of malaria transmission: a review of entomological inoculation rate measurements and methods across sub-Saharan Africa. Malar J. 2009;8:19. doi:10.1186/1475-2875-8-19.

10. Bayoh MN, Mathias DK, Odiere MR, Mutuku FM, Kamau L, Gimnig JE, et al. Anopheles gambiae: historical population decline associated with regional distribution of insecticide-treated bed nets in western Nyanza Province, Kenya. Malar J. 2010;9:62

11. Beier JC, Killeen GF, Githure JI. Short report: entomologic inoculation rates and Plasmodium falciparum malaria prevalence in Africa. Am J Trop Med Hyg. 2009;61:109-13.

12. Wongsrichanalai C, Barcus MJ, Muth S, Sutamihardja A, Wernsdorfer WH. A review of malaria diagnostic tools: microscopy and rapid diagnostic test (RDT). Am J Trop Med Hyg. 2007;77 Suppl 6:119-27.

13. Bousema T, Youssef RM, Cook J, Cox J, Alegana VA, Amran J, et al. Serologic markers for detecting malaria in areas of low endemicity. Emerg Infect Dis. 2010;16:392-9. doi:10.3201/eid1603.090732.

14. Badu K, Afrane YA, Larbi J, Stewart VN, Waitumbi J, Angov E, et al. Marked variation in MSP-119 antibody responses to malaria in western Kenyan highlands. BMC Infect Dis. 2012;12:50. doi:10.1186/1471-2334-12-50.
15. Corran P, Coleman P, Riley E, Drakeley C. Serology: a robust indicator of malaria transmission intensity? Trends Parasitol. 2007;23:575-82. doi:10.1016/ j.pt. 2007. 08. 023.

16. Drakeley CJ, Corran PH, Coleman PG, Tongren JE, Mcdonald SLR, Malima R, et al. Estimating medium- and long-term trends in malaria transmission by using serological markers of malaria exposure. Proc Natl Acad Sci U S A. 2005;102:5108-13.

17. Cook J, Kleinschmidt I, Schwabe C, Nseng G, Bousema T, Corran PH, Riley EM, Drakeley CJ. Serological Markers Suggest Heterogeneity of Effectiveness of Malaria Control Interventions on Bioko Island, Equatorial Guinea. PLoS ONE 6(9): e25137. doi:10.1371/journal.pone.0025137.

18. Drakeley C, Cook J. Potential contribution of sero-epidemiological analysis for monitoring malaria control and elimination: historical and current perspectives. In: David Rollinson D, Hay SI, editors. Advances in Parasitology. Burlington: Academic Press; 2009. p. 299-352.

19. Drakeley CJ, Carneiro I, Reyburn H, Malima R, Lusingu JP, Cox J, et al. Altitude-dependent and -independent variations in Plasmodium falciparum prevalence in northeastern Tanzania. J Infect Dis. 2005;191:1589-98.

20. Badu K, Siangla J, Larbi J, Lawson BW, Afrane Y, Ong'echa J, et al. Variation in exposure to Anopheles gambiae salivary gland peptide (gSG6-P1) across different malaria transmission settings in the western Kenya highlands. Malar J. 2012;11:318.

21. Ribeiro JM, Makoul GT, Levine J, Robinson DR. Spielman A Antihemostatic, antiinflammatory, and immunosuppressive properties of the saliva of a tick, Ixodes dammini. J Exp Med. 1985;161:332-44.

22. Ribeiro JM. Blood-feeding arthropods: live syringes or invertebrate pharmacologists ? Infect Agents Dis. 1995;4(3):143-52.

23. Drame PM, Poinsignon A, Marie A, Noukpo H, Doucoure S, Cornelie S, et al. New Salivary Biomarkers of Human Exposure to Malaria Vector Bites, Anopheles mosquitoes - New insights into malaria vectors, Sylvie Manguin, Editor. ISBN: 978-953-51-1188-7, InTech, DOI:10.5772/55613. http://www. intechopen.com/books/anopheles-mosquitoes-new-insights-into-malariavectors/new-salivary-biomarkers-of-human-exposure-to-malaria-vector-bites

24. Dama E, Cornelie S, Bienvenu M, Camara M, Kambire R, Jamonneau C, et al. Bucheton AMB Identification of Glossina palpalis gambiensis specific salivary antigens: towards the development of a serologic biomarker of human exposure to tsetse flies in West Africa. Microb Infect. 2013;15:416-27.

25. Nascimento RJ, Santana JM, Lozzi SP, Araujo CN, Teixeira AR. Human IgG1 and IgG4: the main antibodies against Triatoma infestans (Hemiptera: Reduviidae) salivary gland proteins. Am J Trop Med Hyg. 2001;65:219-26.

26. Barral A, Honda E, Caldas A, Costa J, Vinhas V, Rowton ED, et al. Human immune response to sand fly salivary gland antigens: a useful epidemiological marker? Am J Trop Med Hyg. 2000;62:740-5.

27. Stone W, Bousema T, Jones S, Gesase S, Hashim R, Gosling R, et al. IgG responses to Anopheles gambiae salivary antigen gSG6 detect variation in exposure to malaria vectors and disease risk. PLoS One. 2012;7(6):e40170. doi:10.1371/journal.pone.0040170.

28. Remoue F, Cisse B, Ba F, Sokhna C, Herve JP, Boulanger D, et al. Evaluation of the antibody response to Anopheles salivary antigens as a potential marker of risk of malaria. Trans R Soc Trop Med Hyg. 2006;100:363-70. doi:10.1016/j.trstmh.2005.06.032.

29. Lombardo F, Ronca R, Rizzo C, Fiorentino G, Bourgouin C. The Anopheles gambiae salivary protein gSG6: an anopheline-specific protein with a bloodfeeding role. Insect Biochem \&Mol Bio. 2009;39:457-66. doi:10.1016/ j.ibmb.2009.04.006.

30. Poinsignon A, Cornelie S, Lanfrancotti A, Rossignol M, Boulanger D, Cisse B, et al. Novel peptide marker corresponding to salivary protein gSG6 potentially identifies exposure to anopheles bites. PLoS One. 2008:3:e2472. doi:10.1371/journal.pone.0002472.

31. Sagna AB, Sarr JB, Gaayeb L, Drame PM, Ndiath MO, Senghor S, et al. gSG6P1 salivary biomarker discriminates micro-geographical heterogeneity of human exposure to Anopheles bites in low and seasonal malaria areas. Parasites \& Vectors. 2013;6:68. doi:10.1186/1756-3305-6-68.

32. Drame PM, Poinsignon A, Besnard P, Cornelie S, Le MJ, Toto JL, et al. Human antibody responses to the Anopheles salivary gSG6-P1 peptide: a novel tool for evaluating the efficacy of ITNs in malaria vector control. PLoS One. 2010;5(12):e15596. doi:10.1371/journal.pone.0015596.

33. Adu B, Dodoo D, Adukpo S, Hedley PL, Arthur FKN, Gerd AT, et al. Fc gamma receptor IIIB (FcCRIIIB) polymorphisms are associated with clinical malaria in Ghanaian children. PLoS One. 2012;7(9):e46197. doi:10.1371/ journal.pone.0046197. 
34. Afari EA, Appawu M, Dunyo S, Baffoe-Wilmot A, Nkrumah FK. Malaria infection, morbidity and transmission in two ecological zones Southern Ghana. Afr J Health Sci. 1995:2:312-5.

35. Akua I, Ansah E, Gyapong M, Adjei S, Barnish G, Evans D. Strategies to improve adherence to recommended chloroquine treatment regimes: a quasi-experiment in the context of integrated primary health care delivery in Ghana. Soc Sci Med. 2002:55:2215-26.

36. Tay SCK, Badu K, Mensah AA, Gbedema SY. The prevalence of malaria among HIV seropositive individuals and the impact of the co- infection on their hemoglobin levels. Ann Clin MicroB Antimicrob. 2015;14:10. doi:10.1186/s12941-015-0064-6.

37. Kusi KA, Bosomprah S, Dodoo D, Kyei-baafour E, Dickson EK, Mensah D, et al. Anti-sporozoite antibodies as alternative markers for malaria transmission intensity estimation. Malar J. 2014:9:317.

38. Tusting LS, Bousema T, Smith DL, Drakeley C. Measuring changes in plasmodium falciparum transmission: precision, accuracy and costs of metrics. Adv Parasitol. 2014;84:151-208. doi:10.1016/B978-0-12-8000991.00003-X

39. Cook J, Reid H, lavro J, Kuwahata M, Taleo G, Clements A, et al. Using serological measures to monitor changes in malaria transmission in Vanuatu. Malar J. 2010;9:169.

40. Drame PM, Diallo A, Poinsignon A, Boussari O, Dos S, Machault V. Evaluation of the effectiveness of malaria vector control measures in urban settings of Dakar by a specific anopheles salivary biomarker. PLoS One. 2013;8(6):e66354. doi:10.1371/journal.pone.0066354.

41. Rizzo C, Ronca R, Fiorentino G, Verra F, Mangano V, Poinsignon A, et al Humoral response to the anopheles gambiae salivary protein gSG6: a serological indicator of exposure to afrotropical malaria vectors. PLoS One. 2011;6:e17980. doi:10.1371/journal.pone.0017980.

42. Orlandi-Pradines E, Almeras L, Denisde Senneville L, Barbe S, Remoué F, Villard C, et al. Antibody response against saliva antigens of Anopheles gambiae and Aedes aegypti in travellers in tropical Africa. Microbes Infect. 2007;9(12-13):1454-62.

43. Ambrosino E, Dumoulin C, Orlandi-Pradines E, Remoue F, Toure-Baldé A, Tall $A$, et al. A multiplex assay for the simultaneous detection of antibodies against 15 Plasmodium falciparum and Anopheles gambiae saliva antigens. Malar J. 2010;9:317. doi:10.1186/1475-2875-9-317.

44. Zhao X, Chen F, Feng Z, Li X, Zhou X. The temporal lagged association between meteorological factors and malaria in 30 counties in south-west China: a multilevel distributed lag non-linear analysis. Malar J. 2014;13:57. doi:10.1186/1475-2875-13-57.

45. Stewart L, Gosling R, Griffin J, Gesase S, Campo J, Hashim R, et al. Rapid assessment of malaria transmission using Age- specific sero-conversion rates. 2009. p. 4. doi:10.1371/journal.pone.0006083.

46. Drame PM, Poinsignon A, Besnard P, Le Mire J, Dos-santos MA, Sow CS, et al. Human antibody response to anopheles gambiae saliva: an immunoepidemiological biomarker to evaluate the efficacy of insecticide-treated nets in malaria vector control. Am J Trop Med Hyg. 2010;83(1):115-21. doi:10.4269/ajtmh.2010.09-0684.

47. Sagna AB, Gaayeb L, Sarr JB, Senghor S, Poinsignon A, Boutouaba-Combe S, et al. Plasmodium falciparum infection during dry season: IgG responses to Anopheles gambiae salivary gSG6-P1 peptide as sensitive biomarker for malaria risk in Northern Senegal. Malar J. 2013;12:301. doi:10.1186/14752875-12-301.

48. Munyekenye OG, Githeko AK, Zhou G, Mushinzimana E, Minakawa N, Yan G. Plasmodium falciparum Spatial Analysis, Western Kenya Highlands. Emerg Infect Dis. 2005;11:1571-7.

49. Smith DL, Guerra CA, Snow RW, Hay SI. Standardizing estimates of the Plasmodium falciparum parasite rate. Malar J. 2007;6:131. doi:10.1186/14752875-6-131.

50. Githeko AK, Ayisi JM, Odada PK, Atieli FK, Ndenga BA, Githure Jl, et al. Topography and malaria transmission heterogeneity in western Kenya highlands: prospects for focal vector control. Malar J. 2006;5:107. doi:10.1186/1475-2875-5-107.

51. Hay SI, Smith DL, Snow RW, Gates M. Measuring malaria endemicity from intense to interrupted transmission. 378. 2008;8:369. doi:10.1016/S14733099(08)70069-0.
52. Corran PH, Cook J, Lynch C, Leendertse H, Manjurano A, Griffin J, et al. Dried blood spots as a source of anti-malarial antibodies for epidemiological studies. Malar J. 2008;7:195. doi:10.1186/1475-2875-7-195.

53. Baum E, Badu K, Molina DM, Liang X, Felgner PL, Yan G. Protein microarray analysis of antibody responses to Plasmodium falciparum in western Kenyan highland sites with differing transmission levels. PLoS One. 2013;8:e82246.

\section{Submit your next manuscript to BioMed Central and take full advantage of:}

- Convenient online submission

- Thorough peer review

- No space constraints or color figure charges

- Immediate publication on acceptance

- Inclusion in PubMed, CAS, Scopus and Google Scholar

- Research which is freely available for redistribution 